\title{
circ_VMA21 protects WI-38 cells against LPS-induced apoptotic and inflammatory injury by acting on the miR-409-3p/KLF4 axis
}

\author{
Qian Wang, Xiaojing Zhang and Dehong Chen \\ Department of Pediatrics, Jinan Maternalty and Child Care Hospital, Jinan, Shandong, China
}

\author{
Highlights \\ 1. circ_VMA21 ameliorates LPS-induced WI-38 cellular apoptosis and inflammation \\ 2. circ_VMA21 targets miR-409-3p and KLF4 is a target of miR-409-3p \\ 3. circ_VMA21 promotes the expression of KLF4 via sponging miR-409-3p
}

\begin{abstract}
Pneumonia is a common inflammatory lung disease. Circular RNA (circRNA) vacuolar ATPase assembly factor (circ_VMA21) has been reported to mitigate the inflammatory injury in WI-38 cells. This study was to investigate the functional mechanism of circ_VMA21. Cell model was established by lipopolysaccharide (LPS) treatment in WI-38 cells. Cell cycle and apoptosis were analyzed by flow cytometry. Cell proliferation was assessed by colony formation and MTT assays. The expression quantification of circ_VMA21, microRNA-409-3p (miR-409-3p) and Kruppel-like transcription factor 4 (KLF4) was performed by qRT-PCR method. The expression of relative protein was detected by Western blot. Inflammatory response was evaluated by ELISA. The target binding was validated by dual-luciferase reporter assay. Cellular analysis indicated that LPS repressed cell cycle and proliferation but induced apoptosis. circ_VMA21 was downregulated in pneumonia samples and LPS-treated WI-38 cells. Functionally, circ_VMA21 assuaged the LPS-induced apoptotic and inflammatory damages. In addition, circ_VMA21 directly targeted miR-409-3p and its function was dependent on the sponge effect on miR-409-3p. Also, KLF4 was a target of miR-409-3p and miR-409-3p inhibitor attenuated LPS-induced cell injury by upregulating KLF4. Moreover, KLF4 was upregulated by circ_VMA21/miR-409-3p axis. These findings suggested that circ_VMA21 relieved the LPS-induced apoptotic and inflammatory injury by the regulation of miR-409-3p/KLF4 axis.
\end{abstract}

Key words: circ_VMA21 - Pneumonia - miR-409-3p - KLF4

\section{Introduction}

Pneumonia is a severe lung disease with acute lower respiratory infection, which can diver acute and chronic comorbidities in other organs of the body (Quinton et al. 2018). Despite the great advancements have been achieved in prevention and treatment of pneumonia, it still remains one of the pri-

Electronic supplementary material. The online version of this article (doi: 10.4149/gpb_2021011) contains Supplementary material.

Correspondence to: Dehong Chen, Department of Pediatrics, Jinan Maternalty and Child Care Hospital, No. 2, Jianguo Xiaojingsan Road, Shizhong District, Jinan, Shandong, 250001, China

E-mail: chdhhf@163.com mary causes of morbidity and mortality in children and older adults (Wardlaw et al. 2006; Petrosillo et al. 2015; le Roux and Zar 2017). Severe inflammation caused by microbial pathogens in pneumonia can lead to the systemic damage (Meijvis et al. 2012). Lipopolysaccharide (LPS) that is the main component of the cell wall of gram-negative bacteria has been considered as an effective stimulator of the inflammatory response in the immune system (Wyns et al. 2015). Genomic techniques have been supposed to improve the therapeutic management and poor prognosis of pneumonia (Gautam et al. 2018).

Circular RNAs (circRNAs) characterized by the covalently closed-loop structures are stable and conservative RNAs in almost all types of eukaryotic cells (Ebbesen et al. 2017). Many studies suggested that circRNAs have pivotal regula- 
tory roles in various kinds of diseases, in various kinds of diseases, such as cancers (Zhao and Shen 2017), cardiovascular diseases (Altesha et al. 2019), neurological diseases (Li et al. 2017) and inflammatory diseases (Ye et al. 2019). Cheng et al. (2018) reported that circRNA vacuolar ATPase assembly factor (circ_VMA21, hsa_circ_0091702) inhibited the inflammatory cytokines-induced intervertebral disc degeneration by regulating the miR-200c/XIAP pathway. Yang et al. (2020) found circ_VMA21 protected against the LPS-induced pneumonia by targeting miR-142-3p.

circRNAs can sponge microRNAs (miRNAs) to further affect the expression level of the downstream genes (Panda 2018). miRNAs are a family of short non-coding RNAs and they can bind to the 3'-untranslated regions (3'-UTRs) of mRNAs (Shahabi et al. 2019). MicroRNA-409-3p (miR-409-3p) was discovered to promote the production of inflammatory cytokines in encephalomyelitis via reducing the SOCS3 expression (Liu et al. 2019b), which was one of the top 3 upregulated miRNAs in pneumonia children (Huang et al. 2018). Kruppel-like transcription factor 4 (KLF4) is an inflammatory palliative in many diseases, such as sepsis ( $\mathrm{Li}$ et al. 2018), atherosclerosis (Hartmann et al. 2016) and cerebral ischemic stroke (Zhang X et al. 2020). Zhang et al. have proved that miR-429 enhanced cell inflammation by downregulating KLF4 in neonatal pneumonia (Zhang L et al. 2020). It remains unknown whether KLF4 is a target of miR-409-3p or circ_VMA21 can regulate the KLF4 expression as a sponge of $\mathrm{miR}-409-3 \mathrm{p}$.

In the present study, we confirmed the target relation between circ_VMA21 or KLF4 and miR-409-3p. A novel mechanism underlying the function of circ_VMA21 in pneumonia injury is disclosed.

\section{Materials and Methods}

\section{Cell culture and LPS treatment}

Human lung fibroblast cell line WI-38 (American Type Culture Collection, ATCC; Manassas, VA, USA) was sustained in Dulbecco's modified Eagle's medium (DMEM; Gibco, Carlsbad, CA, USA) added with heat-inactivated fetal bovine serum (FBS; Gibco, 10\%) and penicillin/streptomycin solution (Gibco, 1\%). Cell culture was conducted in an incubator $\left(37^{\circ} \mathrm{C}\right)$ with humidified air and $5 \% \mathrm{CO}_{2}$. To establish the in vitro model of pneumonia, WI-38 cells were treated with $10 \mu \mathrm{g} / \mathrm{ml}$ LPS (Sigma-Aldrich, St. Louis, MO, USA) for $24 \mathrm{~h}$ and $5 \mu \mathrm{g} / \mathrm{ml}$ LPS was used as a low-concentration control group (Bai et al. 2018).

\section{Cell cycle detection}

Cell cycle was detected using Cell Cycle Assay Kit (Dojindo, Kumamoto, Japan). Cells were stained with propidium iodide
(PI) in accordance with the specification of the manufacturer. Cell distribution was then determined on a flow cytometer (BD Biosciences, San Diego, CA, USA).

\section{Colony formation assay}

200 cells were transplanted into the 6 -well plates and cultured in the incubator $\left(37^{\circ} \mathrm{C}\right)$ for two weeks. Subsequently, colonies were fixated in methanol (Sigma-Aldrich) and stained using crystal violet (Sigma-Aldrich) for $15 \mathrm{~min}$. Then the colonies were photographed and counted.

\section{3-(4,5-dimethylthiazol-2-y1)-2,5-diphenyl tetrazolium bromide (MTT) assay}

Cell proliferation was examined by MTT assay. Cells in each well was incubated with $10 \mu \mathrm{l}$ MTT (Roche, Basel, Switzerland) for $4 \mathrm{~h}$. Formazan was dissolved by $100 \mu \mathrm{l}$ dimethylsulfoxide (DMSO; Invitrogen, Carlsbad, CA, USA), followed by the measurement of the absorbance by a microplate reader (Thermo Fisher Scientific, Waltham, MA, USA) at $570 \mathrm{~nm}$.

\section{Cell apoptosis assay}

The apoptotic cells were stained with Annexin V-fluorescein isothiocyanate (FITC) and PI, in compliance with the instruction book of Annexin V/FITC Apoptosis Detection Kit (Dojindo). By performing the analysis of flow cytometer (BD Biosciences), cells labeled with Annexin V (+)/PI (-) and Annexin V (+)/PI (+) were indicated as the apoptotic cells. Cell apoptotic rate was calculated to evaluate cell apoptosis.

\section{Serum specimens}

31 infantile pneumonia (IP) patients (18 male and 13 female) in this study have not been diagnosed as other diseases. The age range was from $0-6$ years old ( $<2$ years old: $n=5$; $2-4$ years old: $n=17 ; 4-6$ years old: $n=9)$. Peripheral venous blood was collected from IP patients and healthy volunteers $(n=31)$ at Jinan Maternalty and Child Care Hospital. Blood samples were centrifuged at $1200 \mathrm{rpm}$ for $10 \mathrm{~min}$, and the supernatant serum was saved at $-80^{\circ} \mathrm{C}$. The written informed consent forms were signed by the participants or their guardians. This study was authorized by the Ethics Committee of Jinan Maternalty and Child Care Hospital.

\section{The quantitative real-time polymerase chain reaction ( $q R T-P C R)$}

TRIzol $^{\text {TM }}$ Reagent (Invitrogen) was used to extract the total RNA from cells. Reverse transcription was performed using First Strand cDNA Synthesis Kit (Roche). The expres- 
sion level was quantified by KAPA SYBR ${ }^{\oplus}$ FAST One-Step qRT-PCR Master Mix (Roche) and the CFX96 Touch RealTime PCR Detection System (Bio-Rad, Hercules, CA, USA). In addition, RNA stability was assessed by qRT-PCR after treatment of $3 \mathrm{U} / \mu \mathrm{g}$ ribonuclease $\mathrm{R}$ (RNase R; Epicentre Technologies, Madison, WI, USA) in total RNA or $2 \mathrm{mg} / \mathrm{ml}$ actinomycin D (Sigma-Aldrich) in culture medium. The expression analysis was carried out by the $2^{-\Delta \Delta \mathrm{Ct}}$ method. circ_VMA21 (or mRNAs) and miR-409-3p levels were respectively normalized using glyceraldehyde-phosphate dehydrogenase (GAPDH) and U6. The sequences of specific primers were listed as below: circ_VMA21, forward (F): 5'-ACCGTTTTCTGGATGGATTTTG-3' and reverse (R): 5'-GCGACCACTGCAACAATAGC-3'; VMA21, F: 5'-GCATCTACACTGAAGACGCTCC-3' and R: 5'-GACCACTGCAACAATAGCAGCG-3'; miR409-3p, F: 5'-GCGAATGTTGCTCGGTGA-3' and R: 5'-GTGCAGGGTCCGAGGT-3'; KLF4, F: 5'-CATCTCAAGGCACACCTGCGAA-3' and R: 5'-TCGGTCGCATTTTTGGCACTGG-3'; GAPDH, F: 5'-GACTCATGACCACAGTCCATGC-3' and R: 5'-AGAGGCAGGGATGATGTTCTG-3'; U6, F: 5'-CTCGCTTCGGCAGCACA-3' and R: 5'-AACGCTTCACGAATTTGCGT-3'.

\section{Transient transfection}

circ_VMA21 sequence was inserted into the pCD5-ciR overexpression vector (GENESEEO, Guangzhou, China) and the positive vector was named as circ_VMA21. miR409-3p mimic (miR-409-3p) and miRNA mimic (miR-NC), miR-409-3p inhibitor (anti-miR-409-3p) and miRNA inhibitor (anti-miR-NC), small interfering RNA (siRNA) targeting KLF4 (si-KLF4) and siRNA negative control (siNC) were purchased from RIBOBIO (Guangzhou, China). Transient transfection was conducted by Lipofectamine ${ }^{\mathrm{TM}}$ 3000 Transfection Reagent (Invitrogen) following the users' manual.

\section{Western blot}

Protein extraction was performed by RIPA Lysis and Extraction Buffer (Thermo Fisher Scientific). Protein concentration was determined using BCA kit (Thermo Fisher Scientific), and $30 \mu \mathrm{g}$ proteins were applied for the Western blot analysis (Zhang et al. 2017). The primary antibodies were purchased from Cell Signaling Technology (CST, Boston, MA, USA): Ki67 (\#9129, 1:1000), B-cell lymphoma-2 (Bcl-2; \#4223, 1:1000), Bcl-2-associated X (Bax; \#5023, 1:1000), Cleaved-poly-ADP-ribose polymerase (Cleaved-PARP; \#5625, 1:1000), KLF4 (\#12173, 1:1000), Interleukin-6 (IL-6; \#12153, 1:1000), tumor necrosis factoralpha (TNF- $\alpha$; \#6945, 1:1000), GAPDH (\#5174, 1:1000). The secondary antibody Anti-rabbit IgG, HRP (\#7074,
1:2000) was also acquired from CST (SignalFire ${ }^{\mathrm{TM}}$ Plus ECL Reagent). The protein bands were visualized using CST followed by the protein analysis on the ImageLab software version 4.1 (Bio-Rad).

\section{Enzyme-linked immunosorbent assay (ELISA)}

The concentrations of inflammatory cytokines TNF- $\alpha$, IL-6 and IL-8 were examined using Human ELISA Kits (Invitrogen), as per the operating procedures provided by the producer.

\section{Dual-luciferase reporter assay}

circ_VMA21 and KLF4 3'UTR sequences containing the miR-409-3p binding sites were respectively cloned into the pmirGLO vector (Promega, Madison, WI, USA) to construct the circ_VMA21-wt and KLF4-wt plasmids. The miR-409-3p binding sites in circ_VMA21 and KLF4 3'UTR were mutated, then circ_VMA21-mut and KLF4-mut plasmids were obtained by molecular cloning. Cells were plated into the 24-well plates overnight, and co-transfected with the above circ_VMA21 or KLF4 plasmid and miR-409-3p or miR-NC for $48 \mathrm{~h}$. Finally, cell lysate was harvested and the relative luciferase activity was detected using the dual-luciferase reporter assay system (Promega).

\section{Statistical analysis}

Data were shown as the mean \pm standard deviation (SD) and SPSS 24.0 was applied for the statistical analysis. The linear correlation was analyzed using Pearson's correlation coefficient. Student's $t$-test and one-way analysis of variance (ANOVA) followed by Tukey's test were respectively performed to compare the difference between two groups and among multiple groups. $p<0.05$ represented the significant statistical difference.

\section{Results}

LPS inhibited cell cycle progression and proliferation but induced apoptosis in WI-38 cells

After the treatment of LPS, cellular behaviors were analyzed to assess the effects of LPS on WI-38 cells. Cell cycle was blocked from G0/G1 phase to $S$ phase in $10 \mu \mathrm{g} / \mathrm{ml}$ group compared to control and $5 \mu \mathrm{g} / \mathrm{ml}$ groups (Fig. 1A), showing that LPS impeded the progression of cell cycle in WI-38 cells. Colony formation assay (Fig. 1B) and MTT (Fig. 1C) demonstrated that cell proliferation was repressed by LPS. In addition, cell apoptotic rate was elevated in $5 \mu \mathrm{g} / \mathrm{ml}$ and $10 \mu \mathrm{g} / \mathrm{ml}$ LPS groups in comparison 
A

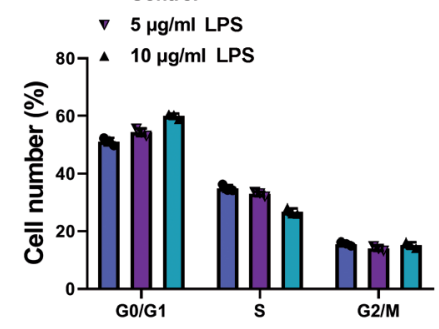

B

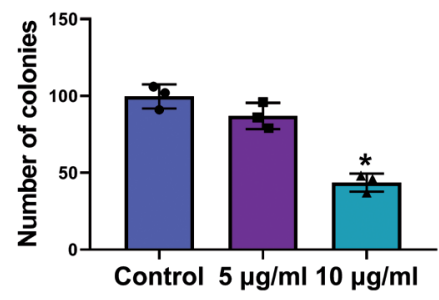

D

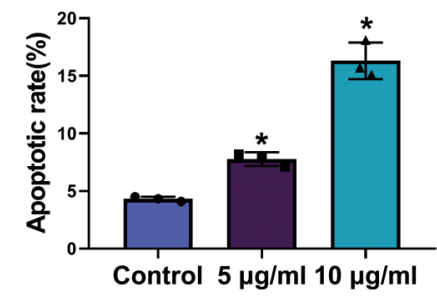

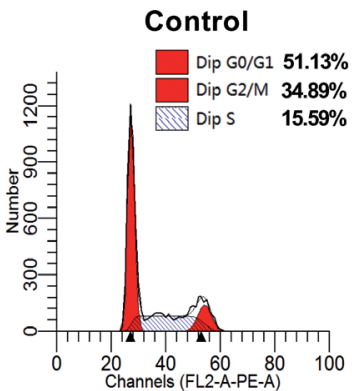
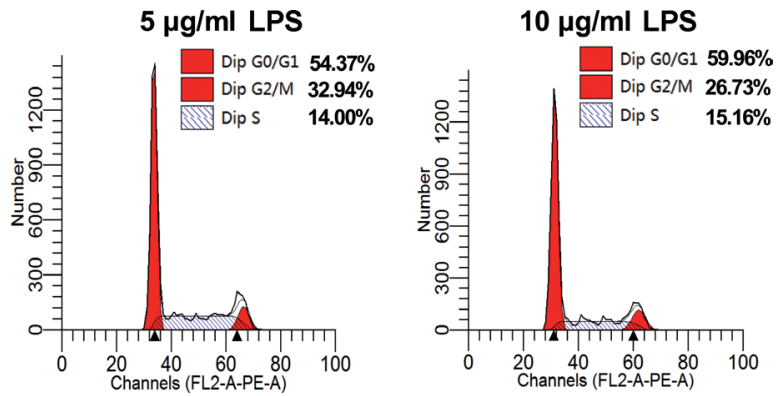

C

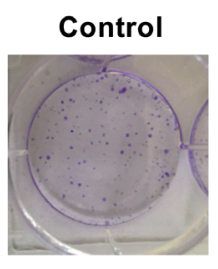

$5 \mu \mathrm{g} / \mathrm{ml}$
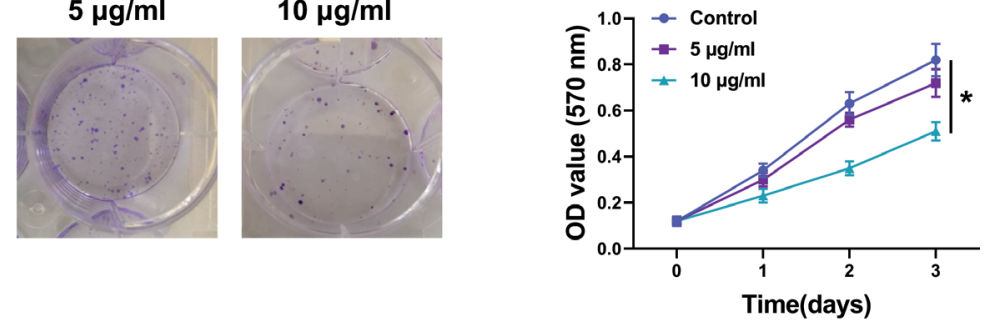

Control

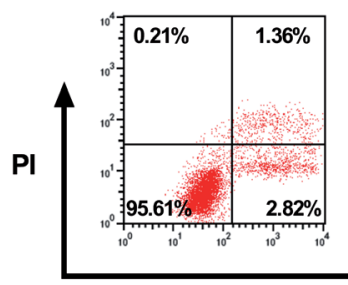

$5 \mu \mathrm{g} / \mathrm{ml}$

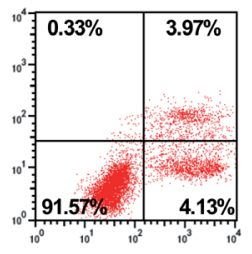

$10 \mu \mathrm{g} / \mathrm{ml}$

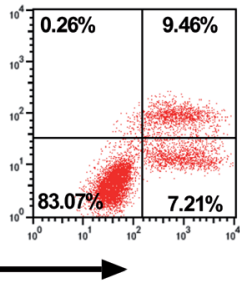

Annexin V-FITC

Figure 1. LPS inhibited cell cycle progression and proliferation, and induced apoptosis in WI-38 cells. WI-38 cells were treated with 5 $\mu \mathrm{g} / \mathrm{ml}$ and $10 \mu \mathrm{g} / \mathrm{ml}$ LPS; untreated cells were used as the control group. A. Cell cycle was detected by flow cytometry. Cell proliferation was determined using colony formation assay (B) and MTT assay (C). D. Cell apoptosis was assessed by flow cytometry. Each experiment was independently performed three times with three technical repetition $(n=3) ;{ }^{*} p<0.05$. LPS, lipopolysaccharide; OD, optical density; PI, propidium iodide; FITC, fluorescein isothiocyanate.

to control group (Fig. 1D). These results indicated that cell injury was successfully induced by LPS in vitro.

\section{circ_VMA21 was downregulated in pneumonia patients and LPS-treated WI-38 cells}

The expression of circ_VMA21 in serum samples and cells was examined using qRT-PCR. As shown in Figure 2A and B, circ_VMA21 expression was significantly declined in IP samples and LPS-treated WI-38 cells contrasted with the normal samples and control cells. circ_VMA21 was found to be resistant to RNase R digestion (Fig. 2C) and actinomycin D treatment (Fig. 2D) while the VMA21 expression was evidently inhibited, which suggested that circ_VMA21 was more stable than the linear VMA21 and it was a circRNA. circ_VMA21 downregulation might be associated with the progression of pneumonia.
circ_VMA21 relieved the LPS-induced apoptosis and inflammation in WI-38 cells

circ_VMA21 vector was constructed to investigate the function of circ_VMA21 in pneumonia. The qRT-PCR manifested that circ_VMA21 transfection upregulated the expression of circ_VMA21 by 9-fold changes while it did not affect the VMA21 level (Fig. 3A). circ_VMA21 vector and control vector were respectively transfected into WI-38 cells under the treatment of $10 \mu \mathrm{g} / \mathrm{ml}$ LPS. It has been observed that the introduction of circ_VMA21 reversed the LPSinduced cell cycle arrest (Fig. 3B), cell proliferation inhibition (Fig. 3C,D) and Ki67 (a proliferation marker) protein downregulation (Fig. 3E). Cell apoptotic rate was lower in LPS+circ_VMA21 group than that in LPS+Vector group (Fig. 3F). Also, the downregulation of anti-apoptotic Bcl-2 but upregulation of pro-apoptotic Bax and Cleaved PARP 

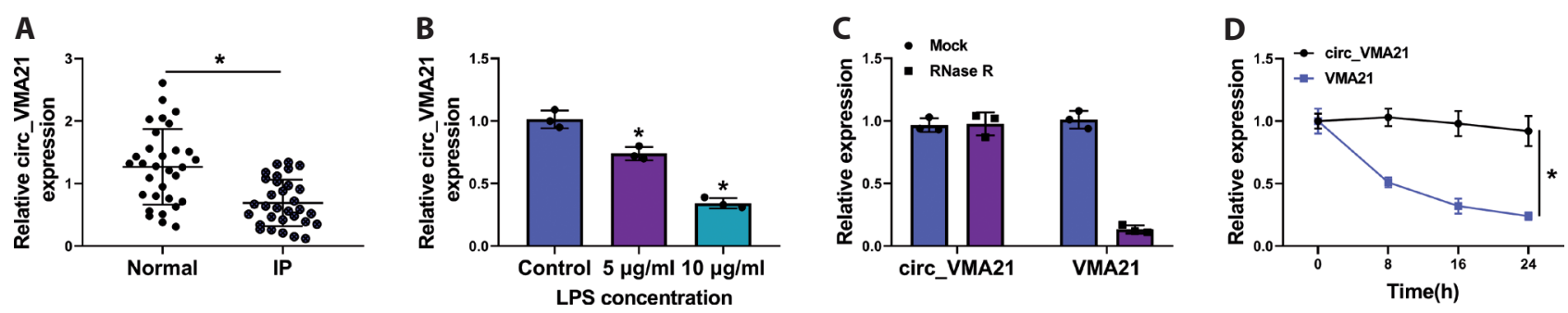

Figure 2. circ_VMA21 was downregulated in pneumonia patients and LPS-treated WI-38 cells. The expression of circ_VMA21 was examined by qRT-PCR in Normal and IP serum samples (A) and control/LPS treatment groups (B). The qRT-PCR was used for the analysis of circ_VMA21 and VMA21 levels after RNase R (C) or actinomycin D (D) treatment $(0,8,16$ and $24 \mathrm{~h})$. Each experiment was independently performed three times with three technical repetition $(n=3) ;{ }^{*} p<0.05$. LPS, lipopolysaccharide; IP, infantile pneumonia; circ_VMA21, circRNA vacuolar ATPase assembly factor.

caused by LPS were mitigated following the transfection of circ_VMA21 (Fig. 3G). Data of ELISA suggested that LPS treatment promoted the concentrations of inflammatory cytokines (TNF- $\alpha$, IL-6 and IL-8), while the overexpression of circ_VMA21 ameliorated this inflammatory response (Fig. 3H). These findings showed that circ_VMA21 could abate the LPS-induced apoptotic and inflammatory injury in WI-38 cells.

circ_VMA21 targeted miR-409-3p

CircBank software was employed to predict the binding sites between circ_VMA21 and miR-409-3p (Fig. 4A). Dual-luciferase reporter assay manifested that miR-409-3p overexpression suppressed the relative luciferase activity of circ_VMA21-wt plasmid while no distinct difference was found in circ_VMA21-mut group (Fig. 4B), affirming that miR-409-3p could bind to circ_VMA21. The qRT-PCR analysis exhibited that miR-409-3p expression was downregulated by the upregulation of circ_VMA21 in WI-38 cells (Fig. 4C). In addition, the level of miR-409-3p was notably upregulated in LPS-treated WI-38 cells (Fig. 4D) and IP serum samples (Fig. 4E) compared to the control groups. Interestingly, a negative relationship $(r=-0.6382, p<0.0001)$ between the expression of circ_VMA21 and miR-409-3p was found in IP serum samples (Fig. 4F). Together, circ_VMA21 could directly target miR-409-3p.

\section{circ_VMA21 lightened the LPS-induced cell injury via sponging miR-409-3p}

The qRT-PCR data displayed that miR-409-3p expression was increased in circ_VMA21+miR-409-3p group in contrast with the circ_VMA21+miR-NC group (Fig. 5A), implying that miR-409-3p was greatly overexpressed by miR409-3p mimic. Subsequently, LPS-treated WI-38 cells were transfected with circ_VMA21, circ_VMA21+miR-409-3p and the corresponding control groups. Functional experi- ments indicated that the effects of circ_VMA21 on cell cycle (Fig. 5B), cell proliferation (Fig. 5C-E) and cell apoptosis (Fig. 5F,G) were abolished after the overexpression of miR409-3p. Meanwhile, ELISA and Western blot indicated that the concentration inhibition (Fig. $5 \mathrm{H}$ ) and protein expression downregulation (Fig. S1A, Supplementary materials) of inflammatory cytokines were also relieved by miR-409-3p mimic. Therefore, the protective function of circ_VMA21 against the LPS-induced cell injury was achieved by sponging miR-409-3p.

\section{KLF4 acted as a target gene for miR-409-3p}

The miRDB analysis presented the complementary sites for miR-409-3p in the 3'UTR sequence of KLF4 (Fig. 6A). The interaction between miR-409-3p and KLF4 was further validated by the dual-luciferase reporter assay (Fig. 6B). The miR-409-3p mimic and anti-miR-409-3p were both efficient on the expression of miR-409-3p by comparison with the miR-NC and anti-miR-NC groups (Fig. 6C). KLF4 mRNA and protein levels were found to be inhibited by miR-409$3 p$ overexpression but increased by miR-409-3p inhibition (Fig. 6D,E). The expression analysis for KLF4 by qRT-PCR and Western blot revealed that it was downregulated in LPStreated WI-38 cells (Fig. 6F,G) and IP serum samples (Fig. $6 \mathrm{H}, \mathrm{I})$. Pearson's correlation coefficient exhibited that there was a negative relation $(r=-0.5496, p=0.0011)$ between miR-409-3p and KLF4 (Fig. 6J) but a positive correlation $(r=0.5392, p=0.0015)$ between circ_VMA21 and KLF4 (Fig. 6K) in IP samples. Altogether, KLF4 was a target of miR-409-3p which related to circ_VMA21.

\section{Downregulation of miR-409-3p weakened cell injury caused by LPS via upregulating the KLF4 expression}

si-KLF4 was used for the knockdown of KLF4 expression. As illustrated in Figure 7A and B, si-KLF4 transfection restored the anti-miR-409-3p-mediated upregulation of KLF4 


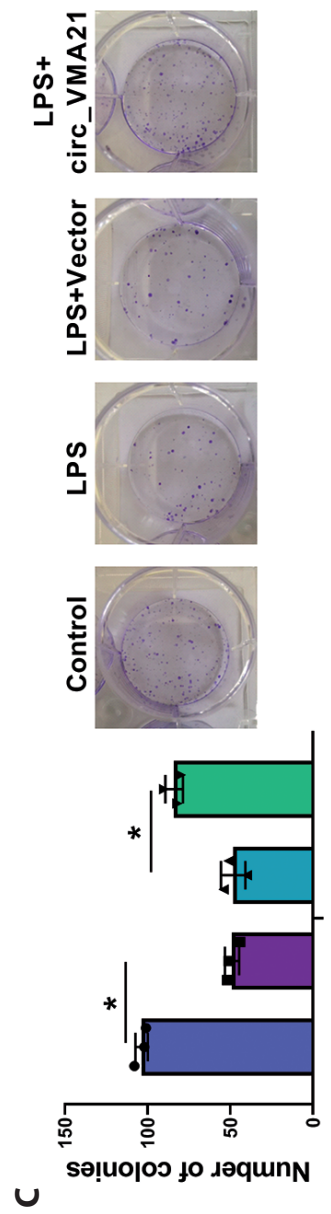

u
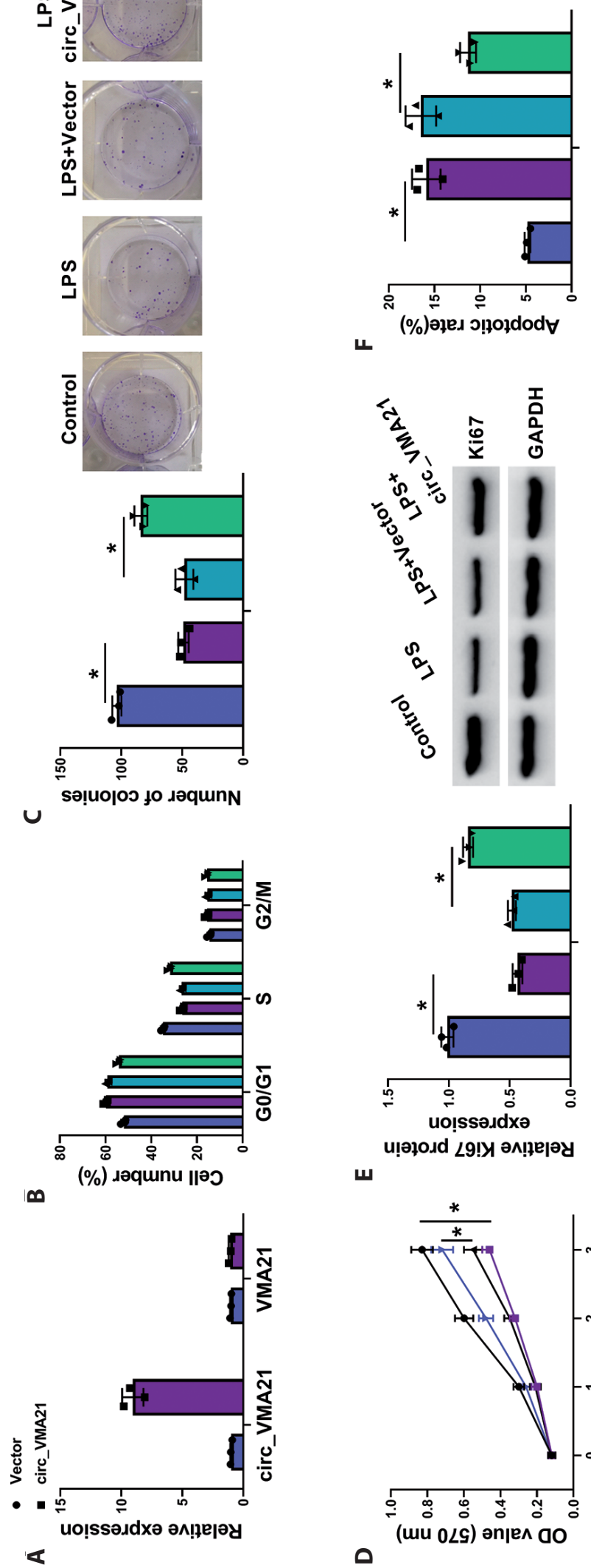

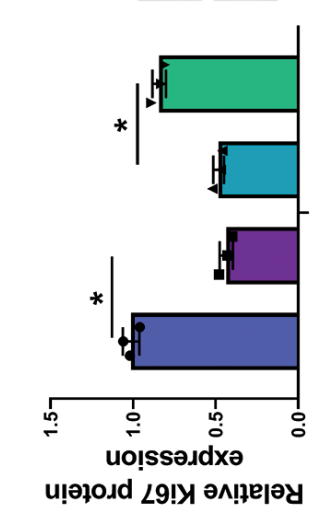

U

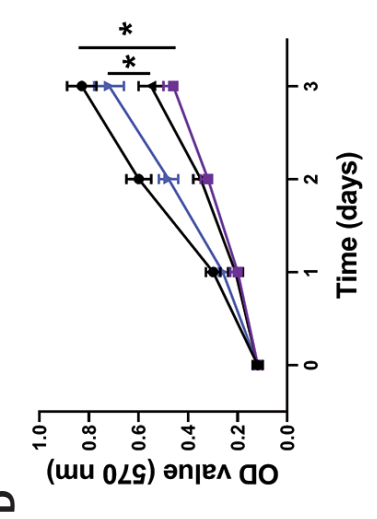

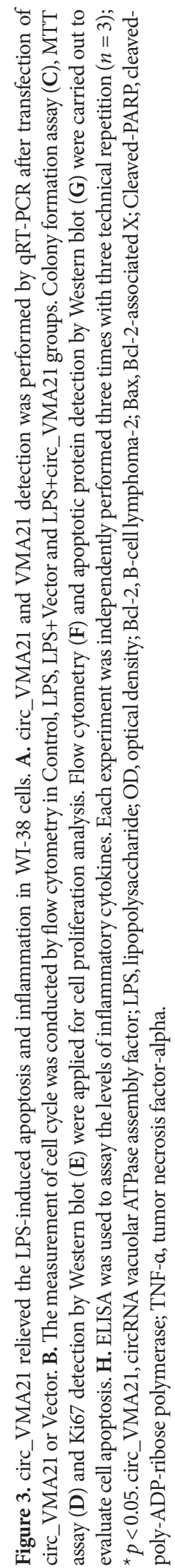


A

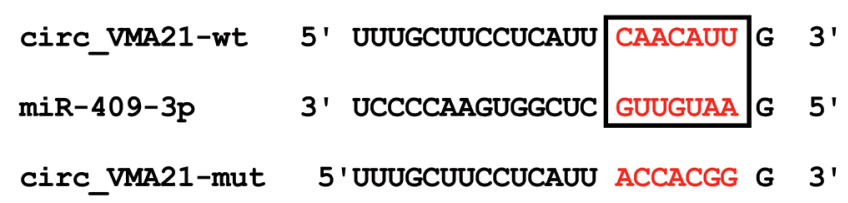

\author{
B
}

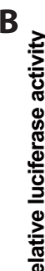

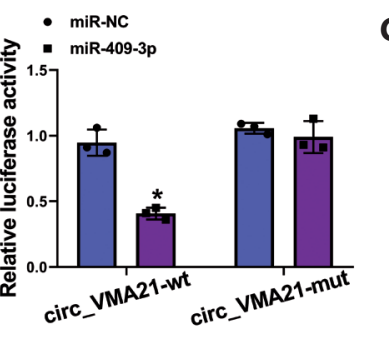

C
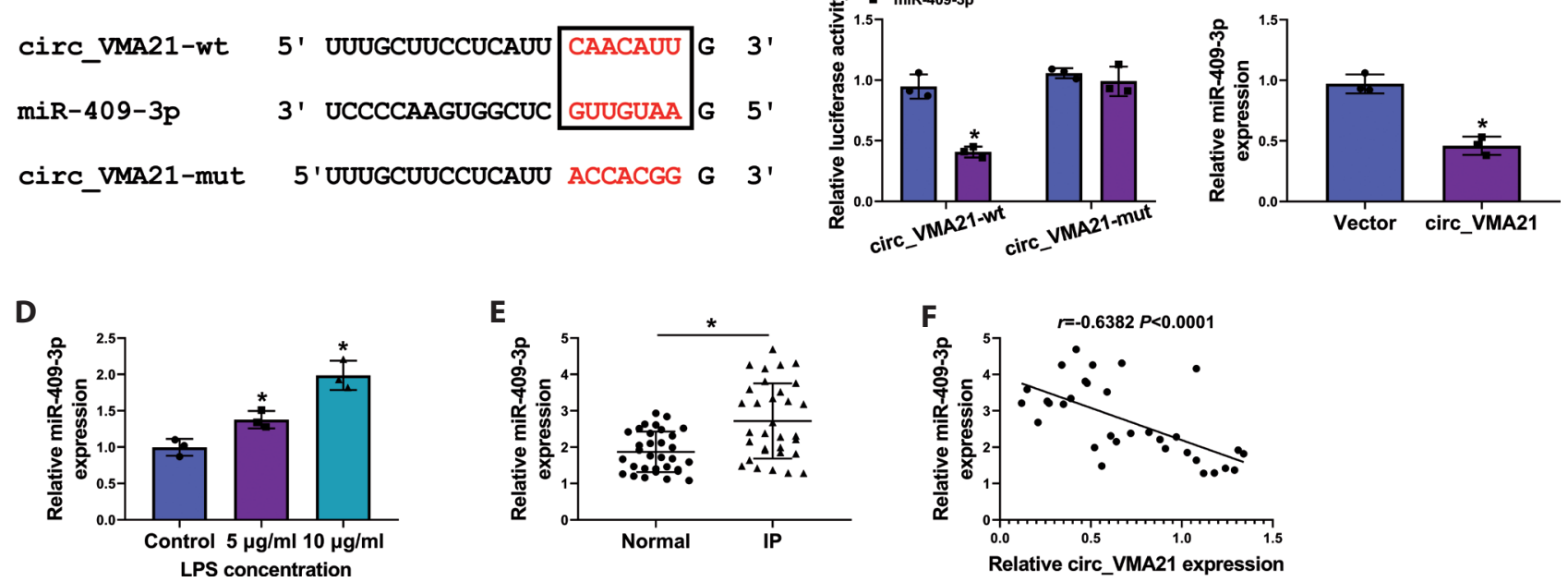

Figure 4. circ_VMA21 targeted miR-409-3p. The binding between circ_VMA21 and miR-409-3p was analyzed by circBank prediction (A) and dual-luciferase reporter assay (B). C. The miR-409-3p expression was determined in Vector and circ_VMA21 transfection groups. The qRT-PCR was used for the expression analysis of miR-409-3p in LPS-treated WI-38 cells (D) and IP samples (E). F. The linear relation between circ_VMA21 and miR-409-3p was analyzed by Pearson's correlation coefficient. Each experiment was independently performed three times with three technical repetition $(n=3) ;{ }^{*} p<0.05$. For abbreviations, see Fig. 2.

mRNA and protein expression. Cell cycle progression was enhanced by miR-409-3p inhibitor in LPS-treated WI-38 cells, which was eliminated by the knockdown of KLF4 (Fig. 7C). Cell proliferation by colony formation assay (Fig. 7D), MTT (Fig. 7E) and Ki67 protein detection (Fig. 7F) was also shown to be facilitated by anti-miR-409-3p transfection, whereas this promotion was reverted following the silence of KLF4 expression. Moreover, flow cytometry and Western blot showed that KLF4 downregulation abrogated the antimiR-409-3p-induced apoptosis inhibition (Fig. 7G,H). Data from ELISA suggested that the downregulation of TNF- $\alpha$, IL- 6 and IL- 8 caused by anti-miR-409-3p was counteracted after KIF4 knockdown in LPS-treated WI-38 cells (Fig. 7I). Consistently, the protein levels of TNF- $\alpha$ and IL- 6 were also upregulated by the introduction of si-KLF4 relative to alone anti-miR-409-3p transfection in LPS-treated cells (Fig. S1B). Taken together, miR-409-3p inhibitor could recede the LPSinduced cell injury via targeting KLF4.

circ_VMA21 upregulated the expression of KLF4 as a sponge of $m i R-409-3 p$

To reveal whether circ_VMA21 could regulate the KLF4 expression via targeting miR-409-3p, reverted transfection (Vector, circ_VMA21, circ_VMA21+miR-NC and circ_VMA21+miR-409-3p) was performed in WI-38 cells. The qRT-PCR and Western blot demonstrated that circ_VMA21 transfection upregulated the mRNA (Fig. $8 \mathrm{~A}$ ) and protein (Fig. 8B) expression of KLF4, which was subsequently returned by miR-409-3p overexpression. Thus, KLF4 could be regulated by the circ_VMA21/ miR-409-3p axis.

\section{Discussion}

Pneumonia is a typical inflammatory disease of human respiratory system. The functional analysis and mechanism exploration in this study confirmed that circ_VMA21 acted as an effective biomarker to inhibit the apoptotic and inflammatory injury in pneumonia by the miR-409-3p/KLF4 axis.

A handful of circRNAs have been revealed to participate in the regulation of pneumonia (Zhao et al. 2019; Zhong et al. 2019). For instance, Jia et al. (2020) stated that circ_406961 inhibited the particulate matter (PM)-induced inflammatory response in human bronchial epithelial $16 \mathrm{HBE}$ cells by interacting with ILF2 and activating the STAT3/JNK pathway. Guo et al. (2020) reported that the silence of circANKRD36 lightened the LPS-aroused cell injury in human embryonic lung fibroblast cells. Knockdown of circ_0038467 remitted the LPS-induced inflammatory damage in $16 \mathrm{HBE}$ cells by regulating the expression of miR-338-3p (Liu et al. 2020), and circZNF652 retarded the progression of pneumonia in WI-38 cells by sponging miR-181 (Liu et al. 2019a). The functional experiments for circ_VMA21 have shown that its overexpression weakened the LPS-triggered cell cycle retardation, proliferation inhibition, apoptotic phenomenon and inflammatory response 


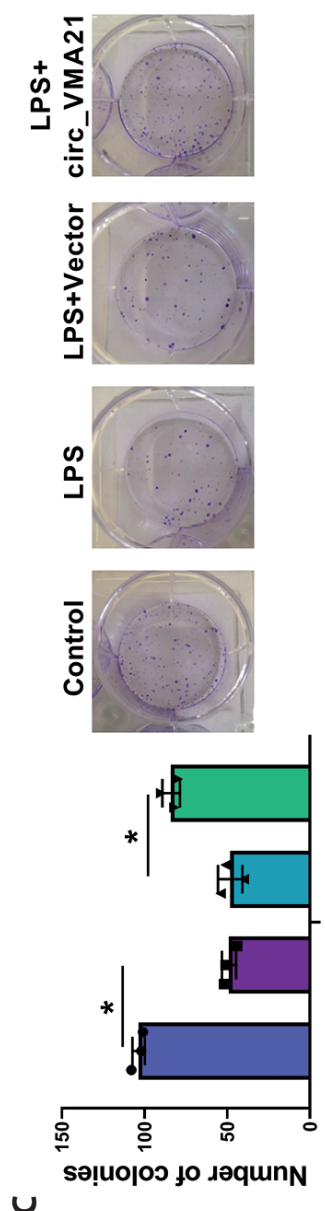

น
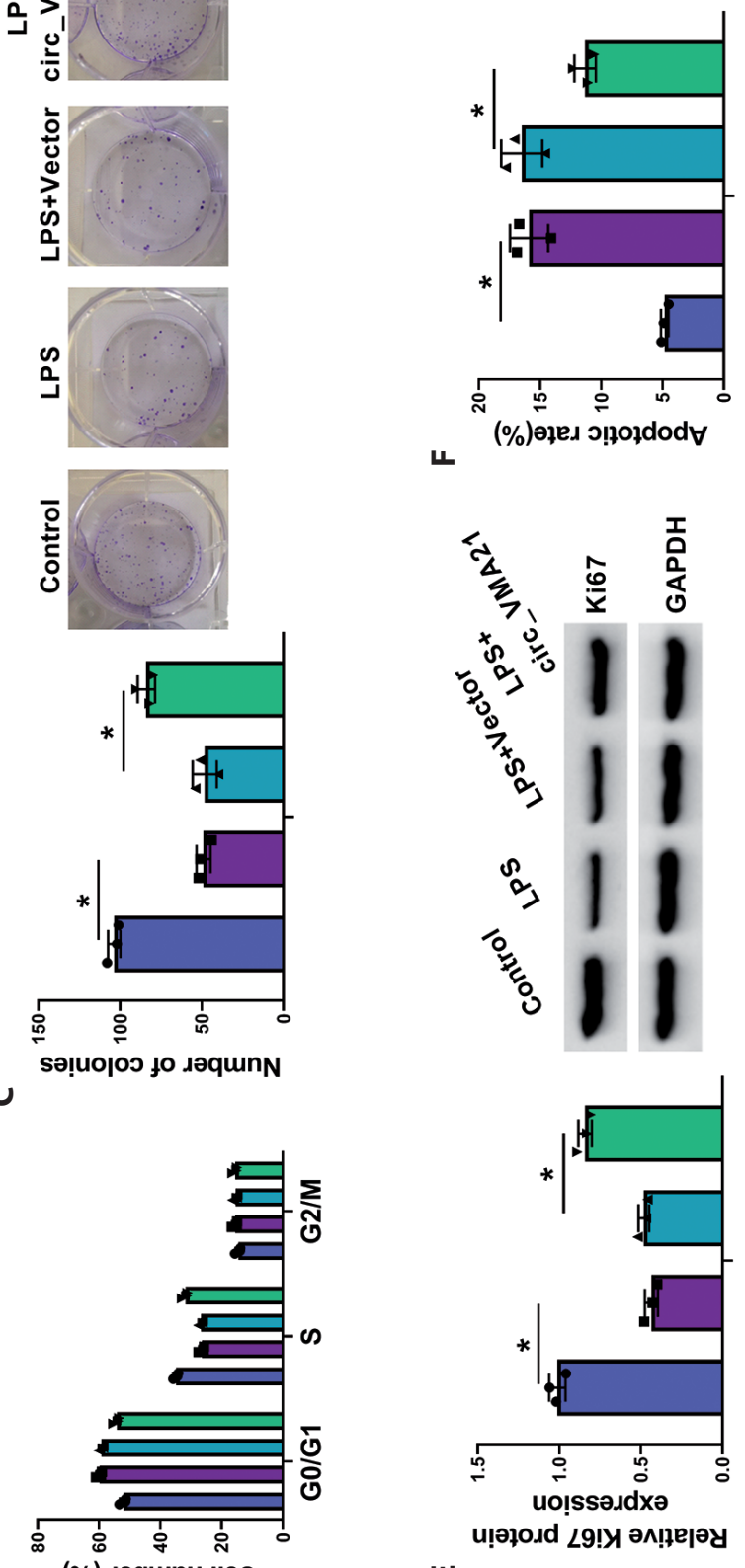

$\infty$

(\%) səqunu ॥əכ

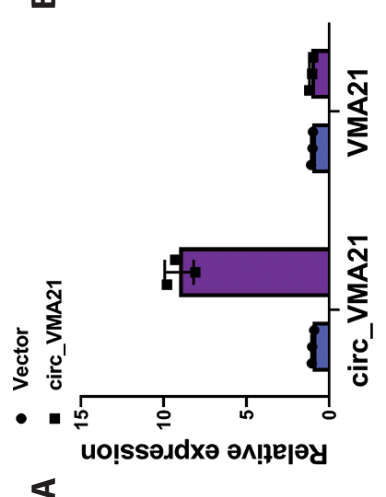

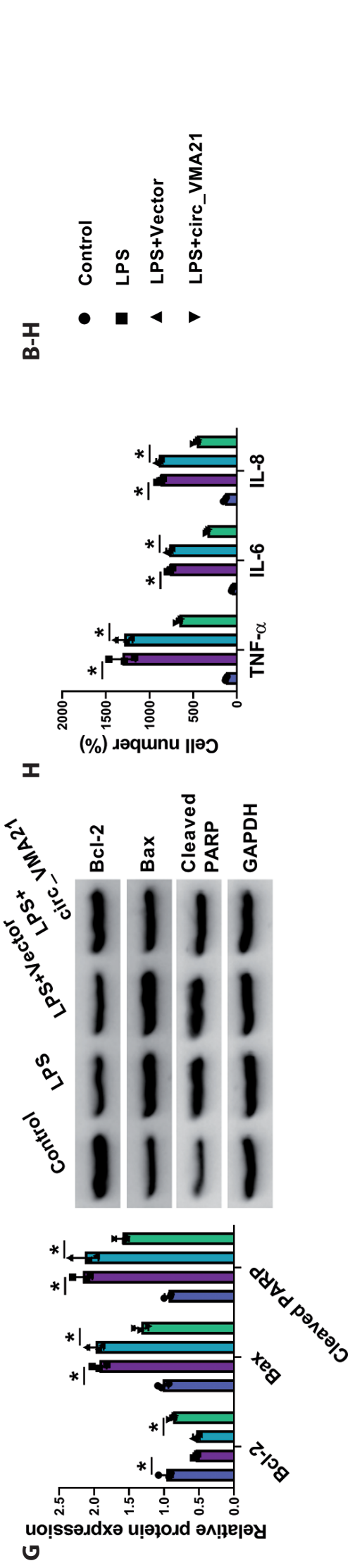



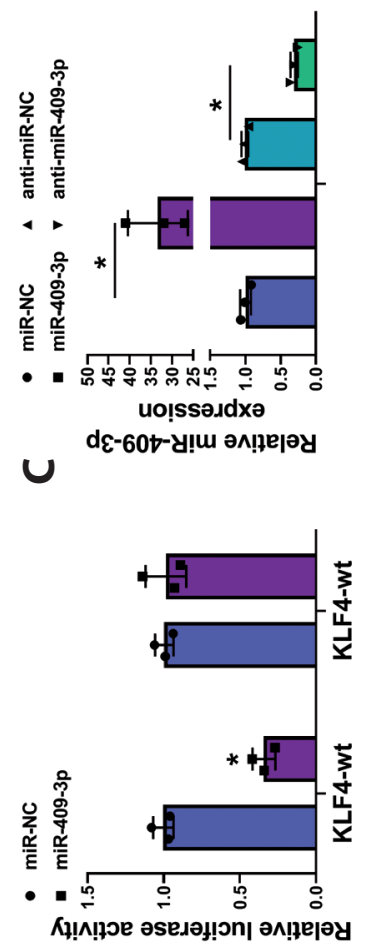

$\infty$

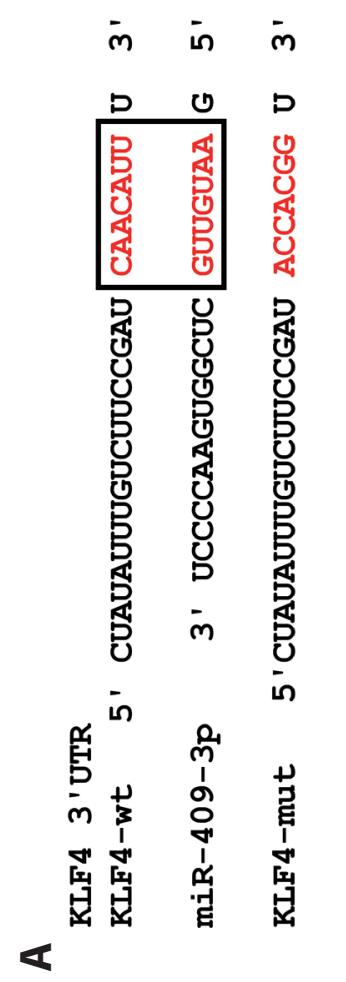

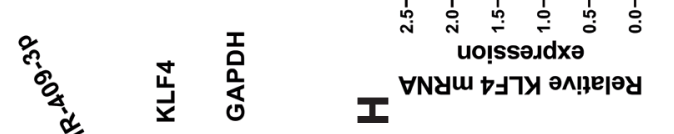
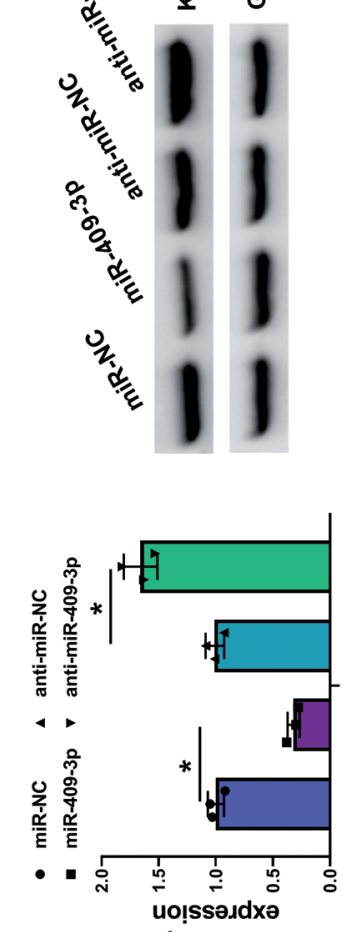

$\mathbf{u}$
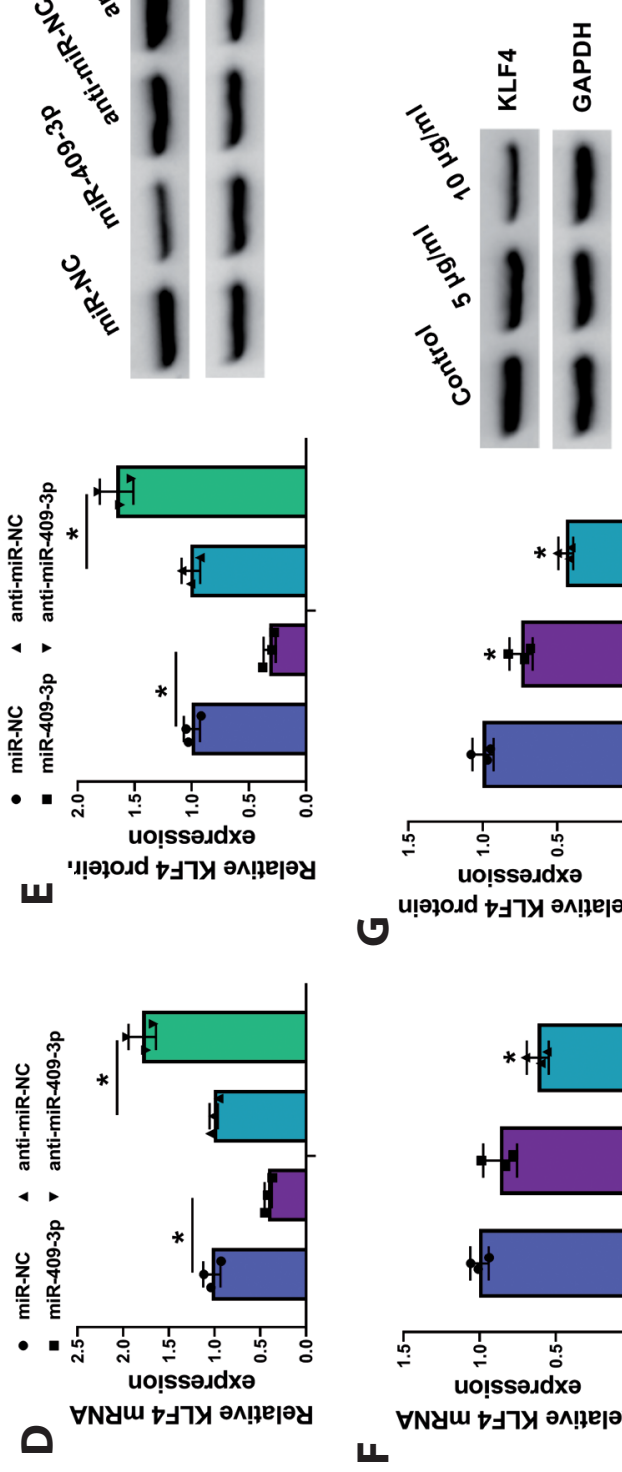

L

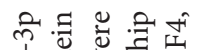

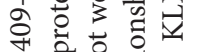

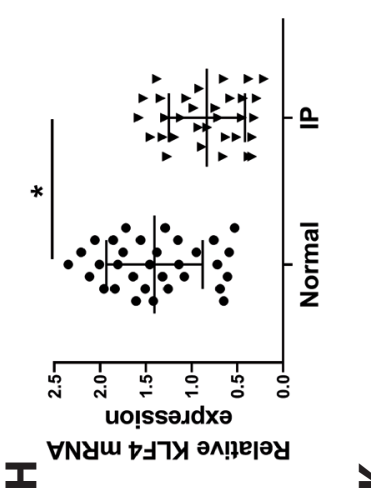

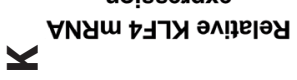
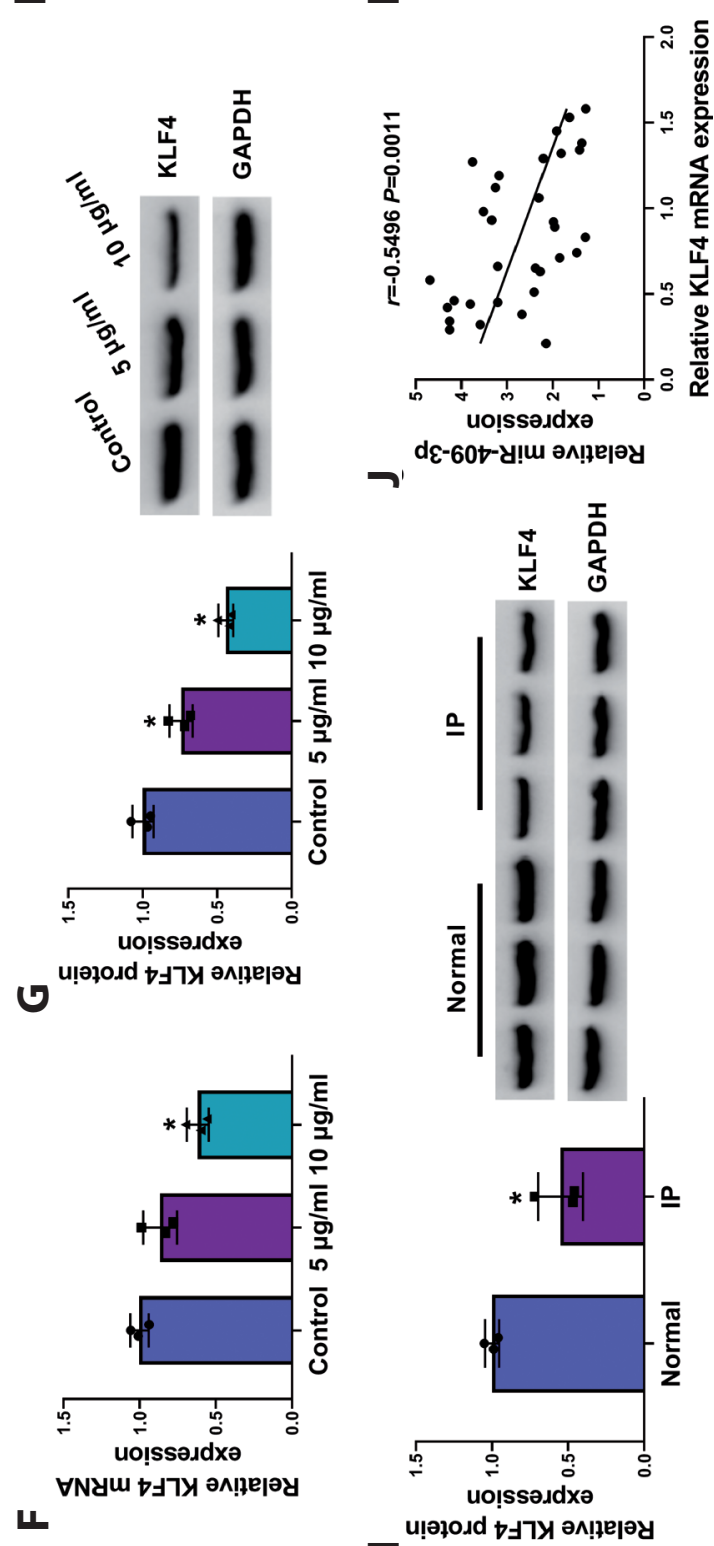

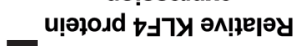

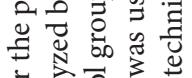

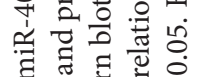

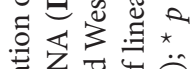

항

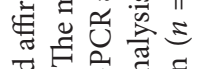

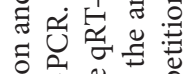

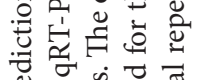

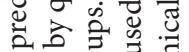

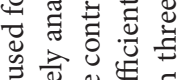

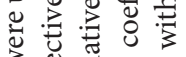

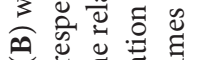

ช

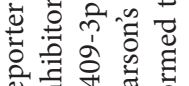

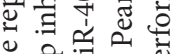

छे के 范

害完䓌氙金

ज्ञ की थे च्च

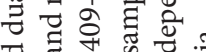

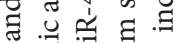

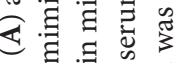

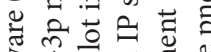

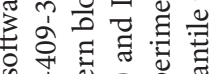

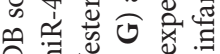

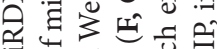

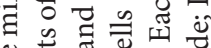

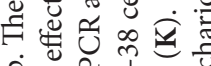

में

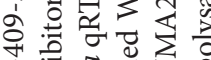

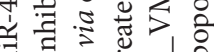

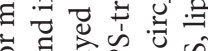

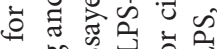

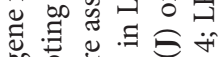

से है के के

छै

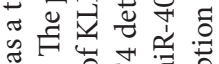

ن ن 出范

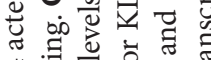

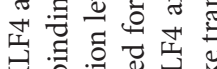

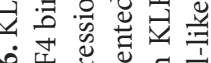

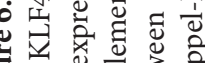

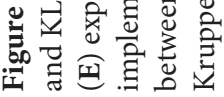

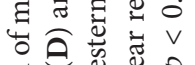

ลे 

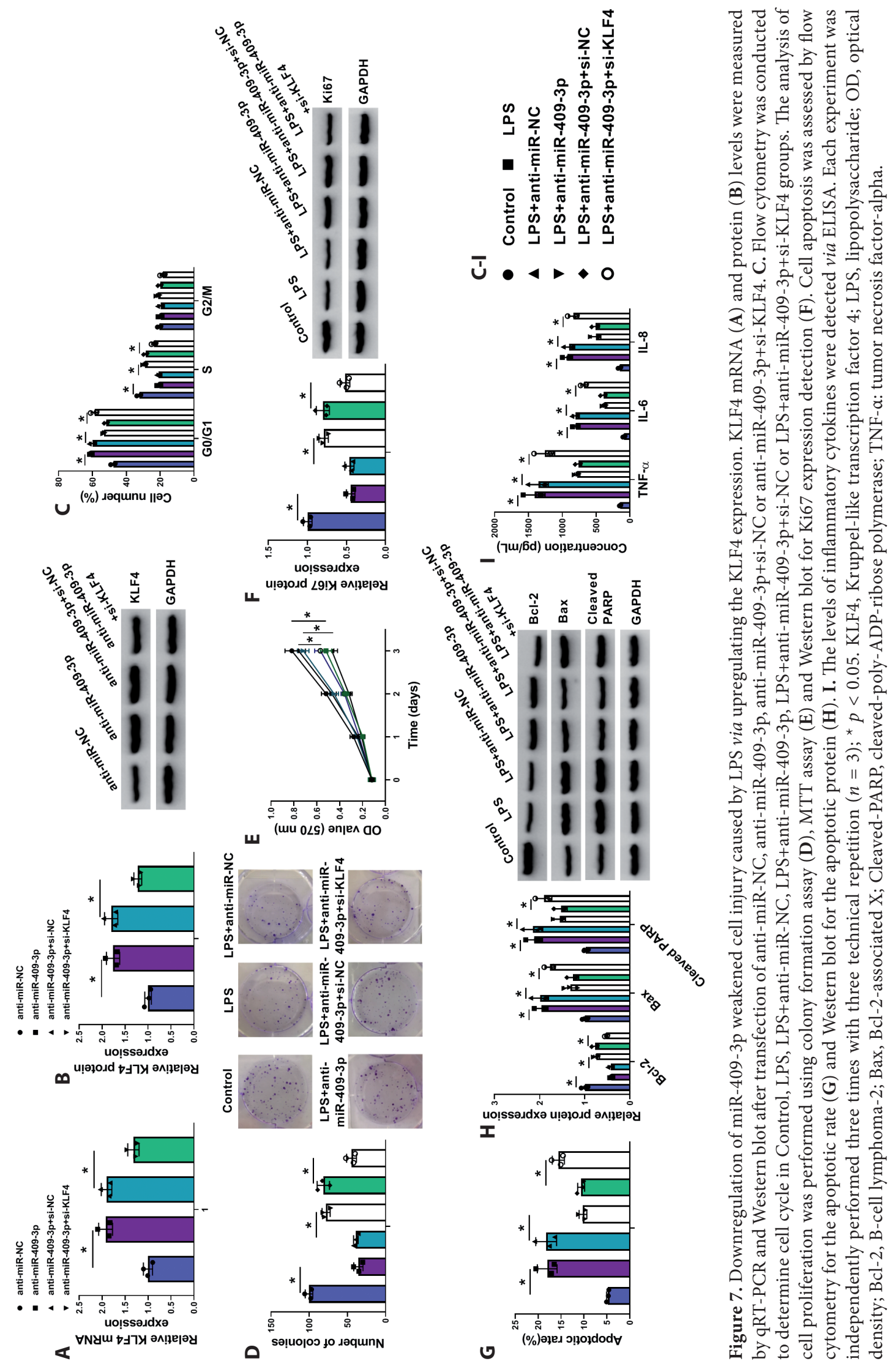

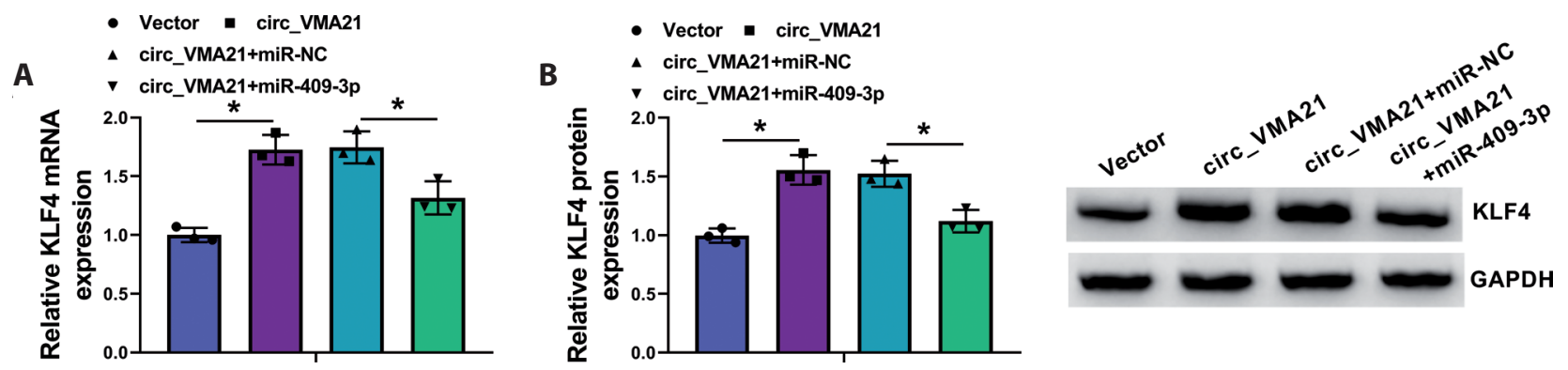

Figure 8. circ_VMA21 upregulated the expression of KLF4 as a sponge of miR-409-3p. The qRT-PCR and Western blot were used to analyze the expression changes of KLF4 after WI-38 cells were transfected with Vector, circ_VMA21, circ_VMA21+miR-NC or circ_VMA21+miR-409-3p. Each experiment was independently performed three times with three technical repetition $(n=3){ }^{*} p<0.05$. circ_VMA21, circRNA vacuolar ATPase assembly factor; KLF4, Kruppel-like transcription factor 4.

in WI-38 cells. circ_VMA21 was identified as an antiapoptotic and anti-inflammatory factor in the pathological injury of pneumonia.

circRNAs are important regulatory molecules in human diseases via acting as the sponges of miRNAs. hsa circ_0000523 repressed cell proliferation and promoted cell apoptosis in colorectal cancer by serving as a miR-31 sponge (Jin et al. 2018). circADAMTS13 could sponge miR-484 to inhibit the proliferation of hepatocellular carcinoma cells (Qiu et al. 2019). circ-ZNF609 has been found to regulate the retinal neurodegeneration by the sponge effect on miR-615 (Wang et al. 2018). Our results manifested that circ_VMA21 combined with miR-409$3 p$ and incurred the negative regulation of miR-409-3p expression. Additionally, the restoration of miR-409-3p for the circ_VMA21-mediated effect suggested that circ_VMA21 attenuated the LPS-induced cell injury by sponging miR-409-3p.

miR-409-3p is a common tumor regulator by targeting different genes. Wu et al. (2019) declared that miR-409-3p repressed osteosarcoma cell proliferation and invasion by downregulating the ZEB1 expression. Zhang et al. (2016) found that miR-409-3p targeted Akt1 to regulate the development of breast cancer. In this study, KLF4 was proved to be a downstream target of miR-409-3p and miR409-3p could regulate the LPS-stimulated WI-38 injury through the negative regulation of KLF4. More interestingly, circ_VMA21 was positively correlated with KLF4 expression in IP serum samples. The expression analysis indicated that circ_VMA21 could sponge miR-409-3p to induce the promoting effect on the KLF4 level. Wang et al. (2020) have shown that circ_0001023 facilitated cell growth and metastasis in gastric cancer by regulating the expression of PHF10 via sequestering miR-409-3p. Herein, circ_VMA21 was considered to alleviate the LPS-induced injury in WI-38 cells by targeting the miR409-3p/KLF4 axis.

\section{Conclusion}

In conclusion, all these results clarified that circ_VMA21 protected WI-38 cells from the LPS-mediated injury by regulating the miR-409-3p/KLF4 axis. The circ_VMA21/miR409-3p/KLF4 molecular network was discovered as a novel pathological mechanism in the progression of pneumonia.

Conflict of interest. The authors declare that they have no financial conflicts of interest.

\section{References}

Altesha MA, Ni T, Khan A, Liu K, Zheng X (2019): Circular RNA in cardiovascular disease. J. Cell. Physiol. 234, 5588-5600 https://doi.org/10.1002/jcp.27384

Bai D, Han A, Cong S (2018): The effect of down-regulation of CCL5 on lipopolysaccharide-induced WI-38 fibroblast injury: a potential role for infantile pneumonia. Iran J. Basic Med. Sci. 21, 449-454

Cheng X, Zhang L, Zhang K, Zhang G, Hu Y, Sun X, Zhao C, Li H, Li YM, Zhao J (2018): Circular RNA VMA21 protects against intervertebral disc degeneration through targeting miR-200c and X linked inhibitor-of-apoptosis protein. Ann. Rheum. Dis. 77, 770-779 https://doi.org/10.1136/annrheumdis-2017-212056

Ebbesen KK, Hansen TB, Kjems J (2017): Insights into circular RNA biology. RNA Biol. 14, 1035-1045 https://doi.org/10.1080/15476286.2016.1271524

Gautam S, Sharma L, Dela Cruz CS (2018): Personalizing the management of pneumonia. Clin. Chest Med. 39, 871-900 https://doi.org/10.1016/j.ccm.2018.08.008

Guo R, Zhang L, Meng J (2020): Circular RNA ANKRD36 attends to lipopolysaccharide-aroused MRC-5 cell injury via regulating microRNA-31-3p. Biofactors 46, 391-401 https://doi.org/10.1002/biof.1592

Hartmann P, Zhou Z, Natarelli L, Wei Y, Nazari-Jahantigh M, Zhu M, Grommes J, Steffens S, Weber C, Schober A (2016): Endothelial Dicer promotes atherosclerosis and vascular in- 
flammation by miRNA-103-mediated suppression of KLF4. Nat. Commun. 7, 10521

https://doi.org/10.1038/ncomms11907

Huang F, Zhang J, Yang D, Zhang Y, Huang J, Yuan Y, Li X, Lu G (2018): MicroRNA expression profile of whole blood is altered in adenovirus-infected pneumonia children. Mediators Inflamm. 2018, 2320640 https://doi.org/10.1155/2018/2320640

Jia Y, Li X, Nan A, Zhang N, Chen L, Zhou H, Zhang H, Qiu M, Zhu J, Ling Y, et al. (2020): Circular RNA 406961 interacts with ILF2 to regulate PM2.5-induced inflammatory responses in human bronchial epithelial cells via activation of STAT3/JNK pathways. Environ. Int. 141, 105755 https://doi.org/10.1016/j.envint.2020.105755

Jin Y, Yu LL, Zhang B, Liu CF, Chen Y (2018): Circular RNA hsa_circ_0000523 regulates the proliferation and apoptosis of colorectal cancer cells as miRNA sponge. Braz. J. Med. Biol. Res. 51, e7811 https://doi.org/10.1590/1414-431x20187811

le Roux DM, Zar HJ (2017): Community-acquired pneumonia in children - a changing spectrum of disease. Pediatr. Radiol. 47, 1392-1398 https://doi.org/10.1007/s00247-017-3827-8

Li TR, Jia YJ, Wang Q, Shao XQ, Lv RJ (2017): Circular RNA: a new star in neurological diseases. Int. J. Neurosci. 127, 726-734 https://doi.org/10.1080/00207454.2016.1236382

Li Z, Jia Y, Han S, Wang X, Han F, Zhang J, Zhang W, Guan H, Hu D (2018): Klf4 alleviates lipopolysaccharide-induced inflammation by inducing expression of MCP-1 induced protein 1 to deubiquitinate TRAF6. Cell. Physiol. Biochem. 47, 2278-2290 https://doi.org/10.1159/000491538

Liu X, Zhao P, Ge W (2019a): Knockdown of circular RNA circZNF652 remits LPS-induced inflammatory damage by regulating miR-181a. Biofactors 46,1031-1040

https://doi.org/10.1002/biof.1606

Liu X, Zhou F, Yang Y, Wang W, Niu L, Zuo D, Li X, Hua H, Zhang B, Kou Y, et al. (2019b): MiR-409-3p and MiR-1896 co-operatively participate in IL-17-induced inflammatory cytokine production in astrocytes and pathogenesis of EAE mice via targeting SOCS3/STAT3 signaling. Glia 67, 101-112 https://doi.org/10.1002/glia.23530

Liu G, Wan Q, Li J, Hu X, Gu X, Xu S (2020): Circ_0038467 regulates lipopolysaccharide-induced inflammatory injury in human bronchial epithelial cells through sponging miR-338-3p. Thorac. Cancer 11, 1297-1308 https://doi.org/10.1111/1759-7714.13397

Meijvis SC, van de Garde EM, Rijkers GT, Bos WJ (2012): Treatment with anti-inflammatory drugs in community-acquired pneumonia. J. Intern. Med. 272, 25-35 https://doi.org/10.1111/j.1365-2796.2012.02554.x

Panda AC (2018): Circular RNAs act as miRNA sponges. Adv. Exp. Med. Biol. 1087, 67-79

https://doi.org/10.1007/978-981-13-1426-1_6

Petrosillo N, Cataldo MA, Pea F (2015): Treatment options for community-acquired pneumonia in the elderly people. Expert Rev. Anti Infect. Ther. 13, 473-485

Qiu L, Huang Y, Li Z, Dong X, Chen G, Xu H, Zeng Y, Cai Z, Liu X, Liu J (2019): Circular RNA profiling identifies circADAMTS13 as a miR-484 sponge which suppresses cell proliferation in hepatocellular carcinoma. Mol. Oncol. 13, 441-455 https://doi.org/10.1002/1878-0261.12424

Quinton LJ, Walkey AJ, Mizgerd JP (2018): Integrative physiology of pneumonia. Physiol. Rev. 98, 1417-1464 https://doi.org/10.1152/physrev.00032.2017

Shahabi A, Naghili B, Ansarin K, Zarghami N (2019): The relationship between microRNAs and Rab family GTPases in human cancers. J. Cell. Physiol. 234, 12341-12352

https://doi.org/10.1002/jcp.28038

Wang JJ, Liu C, Shan K, Liu BH, Li XM, Zhang SJ, Zhou RM, Dong R, Yan B, Sun XH (2018): Circular RNA-ZNF609 regulates retinal neurodegeneration by acting as miR-615 sponge. Theranostics 8, 3408-3415 https://doi.org/10.7150/thno.25156

Wang Y, Zhang J, Chen X, Gao L (2020): Circ_0001023 promotes proliferation and metastasis of gastric cancer cells through miR-409-3p/PHF10 axis. Onco Targets Ther. 13, 4533-4544 https://doi.org/10.2147/OTT.S244358

Wardlaw T, Salama P, Johansson EW, Mason E (2006): Pneumonia: the leading killer of children. Lancet 368, 1048-1050 https://doi.org/10.1016/S0140-6736(06)69334-3

Wu L, Zhang Y, Huang Z, Gu H, Zhou K, Yin X, Xu J (2019): MiR409-3p inhibits cell proliferation and invasion of osteosarcoma by targeting zinc-finger E-box-binding Homeobox-1. Front. Pharmacol. 10, 137 https://doi.org/10.3389/fphar.2019.00137

Wyns H, Plessers E, De Backer P, Meyer E, Croubels S (2015): In vivo porcine lipopolysaccharide inflammation models to study immunomodulation of drugs. Vet. Immunol. Immunopathol. 166, 58-69 https://doi.org/10.1016/j.vetimm.2015.06.001

Yang P, Gao R, Zhou W, Han A (2020): Protective impacts of circular RNA VMA21 on lipopolysaccharide-engendered WI-38 cells injury via mediating microRNA-142-3p. Biofactors 46, 381-390 https://doi.org/10.1002/biof.1593

Ye YL, Yin J, Hu T, Zhang LP, Wu LY, Pang Z (2019): Increased circulating circular RNA_103516 is a novel biomarker for inflammatory bowel disease in adult patients. World J. Gastroenterol. 25, 6273-6288

https://doi.org/10.3748/wjg.v25.i41.6273

Zhang G, Liu Z, Xu H, Yang Q (2016): miR-409-3p suppresses breast cancer cell growth and invasion by targeting Akt1. Biochem. Biophys. Res. Commun. 469, 189-195 https://doi.org/10.1016/j.bbrc.2015.11.099

Zhang J, Hou W, Jia J, Zhao Y, Zhao B (2017): MiR-409-3p regulates cell proliferation and tumor growth by targeting E74-like factor 2 in osteosarcoma. FEBS Open Bio 7, 348-357 https://doi.org/10.1002/2211-5463.12177

Zhang L, Yan H, Wang H, Wang L, Bai B, Ma Y, Tie Y, Xi Z (2020): MicroRNA ( $\mathrm{miR}$ )-429 promotes inflammatory injury by targeting kruppel-like factor 4 (KLF4) in neonatal pneumonia. Curr. Neurovasc. Res. 17, 102-109 https://doi.org/10.2174/1567202617666200128143634

Zhang X, Wang L, Han Z, Dong J, Pang D, Fu Y, Li L (2020): KLF4 alleviates cerebral vascular injury by ameliorating vascular endothelial inflammation and regulating tight junction protein expression following ischemic stroke. J. Neuroinflammation 17, 107 
https://doi.org/10.1186/s12974-020-01780-X

Zhao T, Zheng Y, Hao D, Jin X, Luo Q, Guo Y, Li D, Xi W, Xu Y, Chen Y, et al. (2019): Blood circRNAs as biomarkers for the diagnosis of community-acquired pneumonia. J. Cell. Biochem. 120, 16483-16494 https://doi.org/10.1002/jcb.28863

Zhao ZJ, Shen J (2017): Circular RNA participates in the carcinogenesis and the malignant behavior of cancer. RNA Biol. 14, 514-521 https://doi.org/10.1080/15476286.2015.1122162

Zhong Y, Wang Y, Zhang C, Hu Y, Sun C, Liao J, Wang G (2019): Identification of long non-coding RNA and circular RNA in mice after intra-tracheal instillation with fine particulate matter. Chemosphere 235, 519-526

https://doi.org/10.1016/j.chemosphere.2019.06.122

Received: November 18, 2020

Final version accepted: March 25, 2021 
Supplementary Material

\section{circ_VMA21 protects WI-38 cells against LPS-induced apoptotic} and inflammatory injury by acting on the miR-409-3p/KLF4 axis

\section{Qian Wang, Xiaojing Zhang and Dehong Chen}

Department of Pediatrics, Jinan Maternalty and Child Care Hospital, Jinan, Shandong, China

Supplementary Figures

A

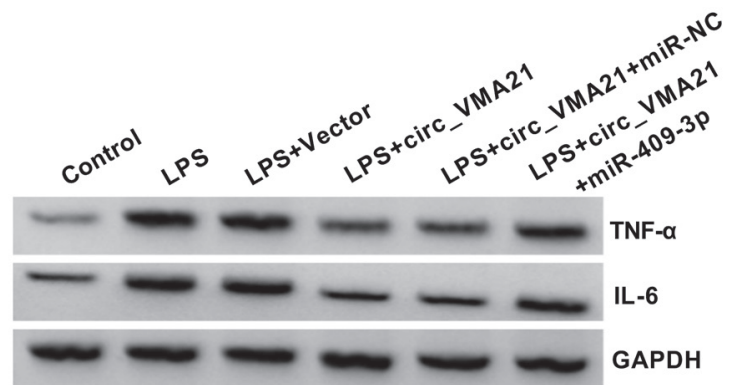

B

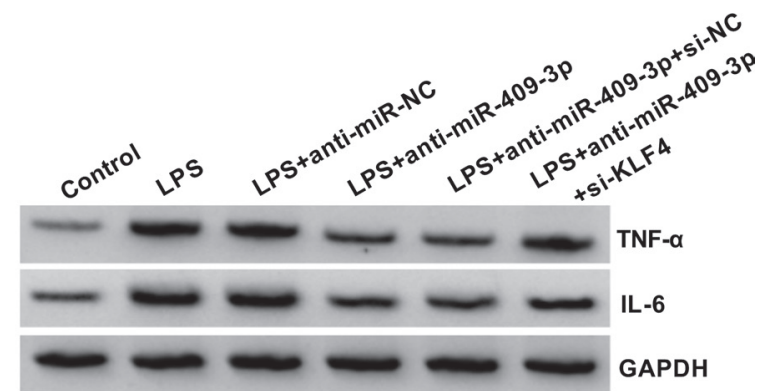

Figure S1. circ_VMA21/miR-409-3p axis and miR-409-3p/KLF4 axis regulated the protein levels of TNF- $\alpha$ and IL-6. The protein detection of TNF- $\alpha$ and IL-6 was performed by Western blot for Fig. 5H (A) and Fig. 7I (B). Each experiment was independently performed three times with three technical repetition $(n=3)$. LPS, lipopolysaccharide; TNF- $\alpha$, tumor necrosis factor-alpha. 